\title{
Generation of a yeast two-hybrid strain suitable for competitive protein binding analysis
}

\author{
Markus Ralser ${ }^{1}$, Heike Goehler ${ }^{2}$, Erich E. Wanker ${ }^{2}$, Hans Lehrach ${ }^{1}$, and \\ Sylvia Krobitsch ${ }^{1}$ \\ ${ }^{1}$ Max Planck Institute for Molecular Genetics and ${ }^{2}$ Max Delbrueck Center for \\ Molecular Medicine, Berlin, Germany
}

BioTechniques 39:165-168 (August 2005)

The yeast two-hybrid (Y2H) system is one of the most common applied genetic systems utilized for the identification of protein-protein interactions in vivo (1). Over the years this system has been systematically exploited for analyzing the physiological function of proteins via the generation of huge protein interaction networks (2). It is based on the principle that different transcription factors can be split into a DNA binding and a polymerase II activation domain. When these independently functional domains are brought into close proximity, the activity of the transcription factor is restored. If an interaction of a protein fused to the DNA binding domain (bait protein) and another protein fused to the activation domain (prey protein) occurs, a functionally transcription factor is reconstructed, and transcription of certain reporter genes involved in amino acid biosynthesis is initiated. The colorimetric reporter $L a c Z$ is often exploited as well, because it allows the relative quantification of its activity and, consequently, the relative strength of protein-protein interactions in vivo (3-5).

Since proteins are multifunctional and interact with many factors, it would be obviously advantageous and in particular less time-consuming to characterize the interplay between proteins in vivo by the $\mathrm{Y} 2 \mathrm{H}$ system in comparison to existing in vitro approaches $(6,7)$. Therefore, the $\mathrm{Y} 2 \mathrm{H}$ technology has been broadened and applied for further characterization of protein-protein interactions. Reverse approaches have been developed allowing the identification of particular protein mutations, peptides, or agents that inhibit the protein-protein interaction of interest $(8,9)$. Furthermore,
Y2H systems have been generated in which the interaction between proteins is enhanced or depends upon expression of a third protein or ligand $(5,10,11)$. However, the simultaneous expression of a third protein or factor to analyze competitive protein binding in the $\mathrm{Y} 2 \mathrm{H}$ background can be difficult, because often the auxotrophic marker genes available have been exhausted in the course of generating the strains for a particular $\mathrm{Y} 2 \mathrm{H}$ system. Moreover, many $\mathrm{Y} 2 \mathrm{H}$ systems are incompatible to each other because of the different promoters and the auxotrophic markers that have to be used. Thus, switching between the available $\mathrm{Y} 2 \mathrm{H}$ systems and strategies is often impossible or very time-consuming due to required cloning efforts for the creation of different bait and prey plasmids needed.

Hence, to facilitate competitive protein binding approaches in a quite frequent $\mathrm{Y} 2 \mathrm{H}$ system, we aimed to create a strain still exploitable for $\mathrm{Y} 2 \mathrm{H}$ screens, but additionally allowing the simultaneous expression of a third protein (Figure 1A). Given that most yeast expression vectors are based on auxothropic markers, we decided to replace the $U R A 3$ gene in the standard strain L40c [MATa his34200 trp1-901 leu2-3,112 ade2 lys2-801am can1

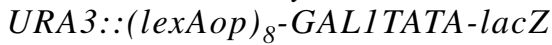


(12) with the Kan $4 M X$ marker gene. For this purpose, the respective DNA fragment was amplified from plasmid pUG6 (13) with primers Kan4MX::ura3 sense and Kan4MX:: ura3 antisense (see Supplementary Table S1 available online at www. BioTechniques.com). Since the yield of the resulting 1.5-kb DNA fragment was very low, we performed a subsequent PCR using this fragment as template and primers $U R A 3$ sense and $U R A 3$ antisense. After transformation (14), recombinants were selected on yeast extract/peptone/dextrose (YPD) media supplemented with $200 \mu \mathrm{g} / \mathrm{mL}$ G418 sulfate (Invitrogen, Carlsbad, CA, USA). Yeast clones were tested for re-growth on YPD/G418 medium and on media lacking uracil to verify the replacement of the URA3 gene. Clones that were deficient for uracil biosynthesis were transformed with bait and prey plasmids encoding interacting proteins and tested for activity of the $L a c Z$ reporter by a $\beta$-galactosidase assay (data not shown). One of the yeast clones that exhibited activity of the $L a c Z$ reporter was selected and named L40KMX. To investigate competitive effects between proteins, we aimed to quantify the relative activity of the colorimetric reporter $L a c Z$ rather than to determine the activation of the auxotrophic markers, because such a growth assay would be biased due to positive selection of yeast that exhibits better growth under the chosen conditions.

For our study, we focused on the interactome of huntingtin (htt) (15), a protein encoded by the ITIS gene in which an expansion of the trinucleotide repeat $\mathrm{CAG}$ is causative for the neurodegenerative disorder Huntington's disease (16). From this network, we selected three proteins that directly interact with huntingtin: (i) the transcription factor CA150 (17); (ii) the actin cytoskeleton organization regulating protein PFN2 (15); and (iii) the formin binding protein HYPA (15). Furthermore, we generated plasmids for expression of huntingtin exon 1 protein (HD1) and endophilin-A3, another interaction partner of huntingtin (12), to analyze their potential competitive effects. Since the bait and prey proteins are expressed under the control of the $A D H 1$ promoter, the proteins tested for competition were placed under the control of the GPD promoter to minimize the risk of promoter effects. The generation of expression plasmids is outlined in supplementary Table S1, while plasmids encoding the prey proteins have been described earlier (15). After transformation of strain L40KMX with the relevant bait and prey plasmids, as well as with expression plasmids encoding either 
HD1 (p426-htt) or endophilin-A3 (p426-EndA3), transformants were selected on media lacking amino acids tryptophan, leucine, and uracil. As controls, we used plasmids p426GPD (empty vector) or p426-GFP encoding the green fluorescent protein (GFP).

For measurements of the LacZ reporter activity, four yeast colonies of each transformation were grown overnight. Then, the same number of cells was transferred into fresh media and grown to an $\mathrm{A}_{600}$ of 0.6-1.0. Yeast cells were lysed by freezing/thawing cycles and resuspended in buffer containing $100 \mathrm{mM}$ HEPES/KOH, $\mathrm{pH}$ 7.0, $150 \mathrm{mM} \mathrm{NaCl}, 2 \mathrm{mM} \mathrm{MgCl} 2,1 \%$ bovine serum albumin (BSA) supplied with $55 \mu \mathrm{L}$ chloroform and $55 \mu \mathrm{L}$ $0.1 \%$ sodium dodecyl sulfate (SDS). After intensive mixing, $500 \mathrm{ng}$ orthonitrophenyl- $\beta$-D-galactopyranoside (ONPG; Applichem GmbH, Darmstadt,
Germany) were added to each lysate, and samples were incubated at $37^{\circ} \mathrm{C}$. After addition of $400 \mu \mathrm{L} 1 \mathrm{M} \mathrm{Na}_{2} \mathrm{CO}_{3}$, samples were centrifuged, and $\mathrm{A}_{420}$ was measured for each sample. The relative $\beta$-galactosidase activity in Miller Units was calculated by the formula $\left[\mathrm{U}_{\mathrm{R}}=\right.$ $\left.\left(1000 \times \mathrm{A}_{420}\right) /\left(\mathrm{vol}_{(\mathrm{mL})} \times \mathrm{t}_{(\min )} \times \mathrm{A}_{600}\right)\right]$ (18). The standard deviation was calculated from four measurements, and the Nalimov method was used with an offset of 1.982 to eliminate outliers with statistical probability larger than 99.9\% (19). In addition, each competitive binding experiment was repeated twice.

As Figure 1B shows, the relative activity of the $L a c Z$ reporter was greatly decreased in yeast expressing lexAHD1 and AD-PFN2 in the presence of HD1 or endophilin-A3, respectively, indicating a strong competitive effect of HD1 and endophilin-A3, respec- tively. A weaker competitive effect was observed in yeast expressing lexA-HD1 and AD-HYPA or lexAHD1 and AD-CA150, respectively. Here, the expression of endophilin-A3 and $\mathrm{HD} 1$, respectively, did not result in a strong reduction of $L a c Z$ reporter gene activity, suggesting that the competitive effect for both proteins is weaker as detected in the case of the interacting pair lexA-HD1 and ADPFN2. However, the competitive effect of endophilin-A3 on the bait/prey pair lexA-HD1 and AD-CA150 cannot be regarded as significant due to the calculated standard deviation. Since the measurement of $L a c Z$ reporter gene activity has often been exploited to determine the relative strength of protein-protein interactions in yeast, we noticed that the interference of the third protein correlated with the relative strength of the protein-protein

A

$B$
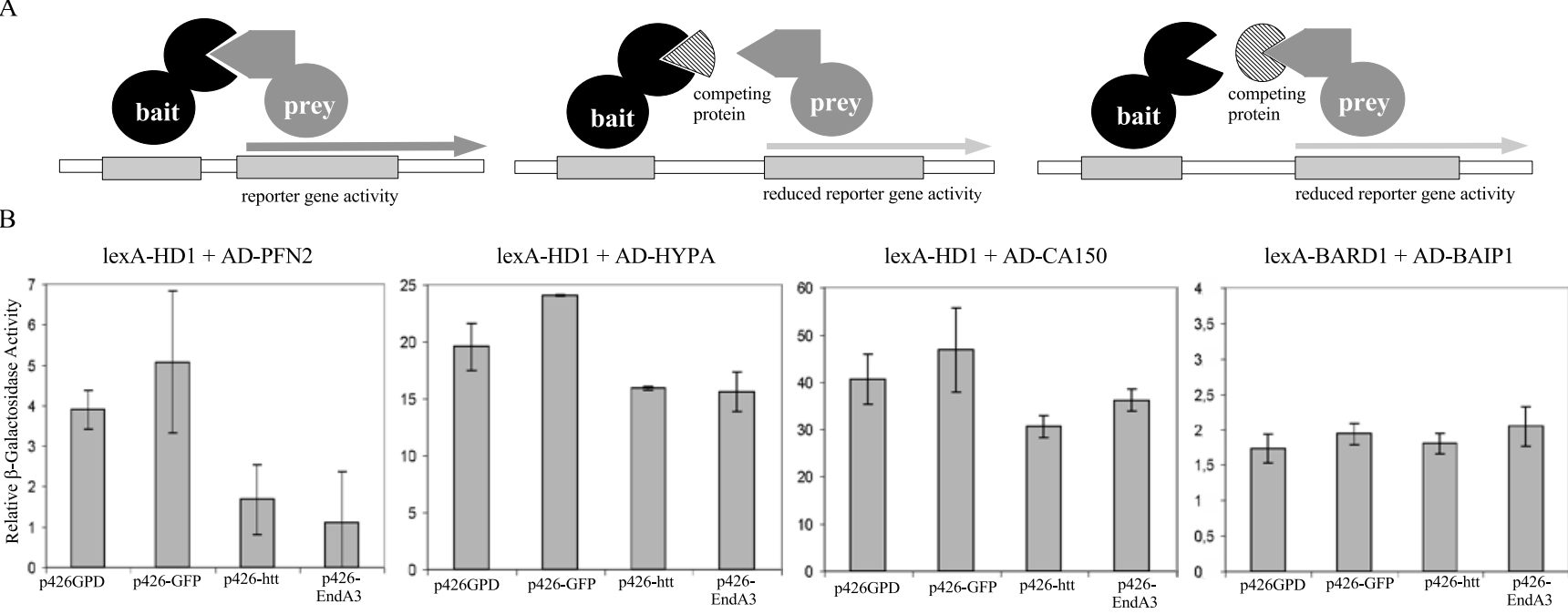

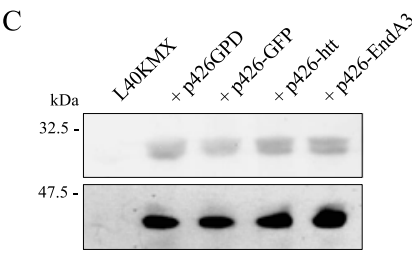

lexA-HD1 + AD-PFN2

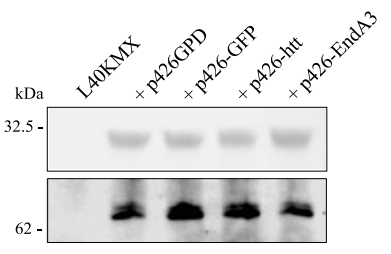

lexA-HD1 + AD-HYPA

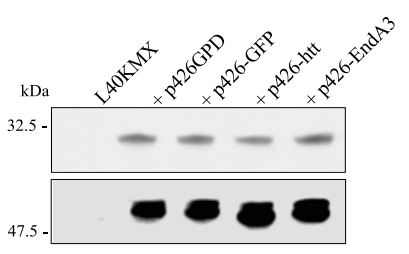

lexA-HD1 + AD-CA150

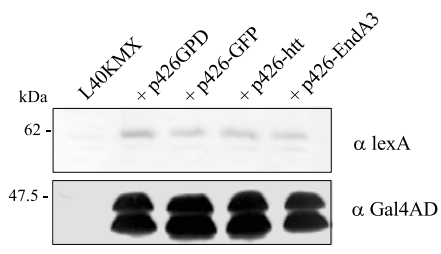

lexA-BARD1 + AD-BAIP1

Figure 1. Applying the yeast two-hybrid (Y2H) system for competitive protein binding analysis. (A) Illustration of the basic principle of the Y2H system. An interaction of bait and prey protein leads to the activation of reporter genes (left panel). If a third factor competes for binding the bait (middle panel) or prey protein (right panel), the expression level of reporter genes is reduced. (B) Competitive protein binding effects on LacZ activity. Strain L40KMX was transformed with the corresponding plasmids as indicated, and the relative activity of the Lac $Z$ reporter was measured as described. Error bars indicate standard deviations. (C) Expression levels of bait and prey proteins. Yeast were grown to an $\mathrm{A}_{600}$ of $0.6-1.0$, and ethanol lysates were prepared. Cell lysates were separated by $10 \%$ sodium dodecyl sulfate polyacrylamide gel electrophoresis (SDS-PAGE) and transferred onto nitrocellulose membranes (Schleicher \& Schuell, Keene, NH, USA). After blocking, the membranes were incubated with $\alpha$-lexA serum (1:5000) or $\alpha$-Gal4AD serum (1:10,000). The polyclonal antibodies against lexA or Gal4AD were generated by injecting HIS $_{6}$-tagged lexA (amino acids 1-202) or glutathione S-transferase (GST)tagged Gal4AD (amino acid 768-881), respectively, into a rabbit. Afterwards, the membranes were incubated with alkaline phosphatase (AP)-conjugated (lexA) or peroxidase-conjugated (Gal4AD) anti-rabbit immunoglobulin G (IgG; Sigma, St. Louis, MO, USA). Proteins were visualized using nitroblue tetrazolium (NBT)/X-phosphate or Western Lightning ${ }^{\mathrm{TM}}$ chemiluminescence reagent (PerkinElmer Life Sciences, Boston, MA, USA). 
interaction. However, the $L a c Z$ reporter gene activity cannot be correlated with the absolute binding affinity between interacting proteins (4). Moreover, we observed that expression of the unrelated GFP protein did not reduce LacZ activity in comparison to the control (empty vector), demonstrating that GFP expression did not affect interactions between the respective bait/prey pairs. To further exclude that the reduction in the relative activity of $L a c Z$ is not a general artifact, but is specifically due to competition of HD1 or endophilin-A3, respectively, we have also selected the protein pair BRCA1associated RING domain protein 1 (BARD) and BARD interacting protein 1 (BAIP1) from the huntingtin network. Both proteins do not directly interact with huntingtin or endophilin-A3 (15). No competitive effect of HD1 or endophilin-A3 was observed in yeast expressing lexA-BARD1 and ADBAIP1. Furthermore, to verify that the differences of $L a c Z$ reporter activity did not result from a reduced expression of the respective bait and prey proteins, we analyzed their intracellular level by immunoblotting (Figure 1C). In comparison to controls, no alterations in expression levels of bait and prey proteins were detected in the presence of additional expressed HD1 or endophilin-A3. Additionally, we tested the stability of the different plasmids by spotting dilution series of the various yeast transformants onto plates lacking the amino acids tryptophan, leucine, or uracil, respectively, to exclude that loss of expression plasmids had occurred. No discrepancy in colony number was observed between the different transformants (data not shown).

Taken together, our data show that the differences of $L a c Z$ reporter activity of the respective yeast clones resulted from competitive binding of HD1 or endophilin-A3 and, consequently, clearly demonstrated that this modified Y2H strain can be exploited for analyzing competitive protein binding in vivo. Although similar Y2H strategies exist, the created strain L40KMX represents a novel analytical tool for directly investigating protein-protein interactions and their interplay in a standard, frequently used $\mathrm{Y} 2 \mathrm{H}$ system.

\section{ACKNOWLEDGMENTS}

We thank A. Ehrenhofer-Murray (Max Planck Institute for Molecular Genetics, Germany) and M. Breitenbach (University of Salzburg, Austria) for providing yeast strains and plasmids. We are grateful to the Max Planck Society for funding part of the research.

\section{COMPETING INTERESTS STATEMENT}

The authors declare no competing interests.

\section{REFERENCES}

1. Fields, S. and O. Song. 1989. A novel genetic system to detect protein-protein interactions. Nature 340:245-246.

2.Xia, Y., H. Yu, R. Jansen, M. Seringhaus, S. Baxter, D. Greenbaum, H. Zhao, and $M$. Gerstein. 2004. Analyzing cellular biochemistry in terms of molecular networks. Annu. Rev. Biochem. 73:1051-1087.

3. Serebriiskii, I.G. and E.A. Golemis. 2000. Uses of lacZ to study gene function: evaluation of beta-galactosidase assays employed in the yeast two-hybrid system. Anal. Biochem. 285:1-15.

4. Estojak, J., R. Brent, and E.A. Golemis. 1995. Correlation of two-hybrid affinity data with in vitro measurements. Mol. Cell. Biol. 15:5820-5829.

5. McAlister-Henn, L., N. Gibson, and E. Panisko. 1999. Applications of the yeast twohybrid system. Methods 19:330-337.

6. Piehler, J. 2005. New methodologies for measuring protein interactions in vivo and in vitro. Curr. Opin. Struct. Biol. 15:4-14.

7. Vikis, H.G. and K.L. Guan. 2004. Glutathione-S-transferase-fusion based assays for studying protein-protein interactions. Methods Mol. Biol. 261:175-186.

8. Vidal, M., R.K. Brachmann, A. Fattaey, E. Harlow, and J.D. Boeke. 1996. Reverse two-hybrid and one-hybrid systems to detect dissociation of protein-protein and DNA-protein interactions. Proc. Natl. Acad. Sci. USA 93:10315-10320.

9. Vidal, M., P. Braun, E. Chen, J.D. Boeke, and E. Harlow. 1996. Genetic characterization of a mammalian protein-protein interaction domain by using a yeast reverse two-hybrid system. Proc. Natl. Acad. Sci. USA 93:10321-10326.

10.Tirode, F., C. Malaguti, F. Romero, R. Attar, J. Camonis, and J.M. Egly. 1997. A conditionally expressed third partner stabilizes or prevents the formation of a transcriptional activator in a three-hybrid system. J. Biol. Chem. 272:22995-22999.

11.Van Aelst, L., M. Barr, S. Marcus, A. Polverino, and M. Wigler. 1993. Complex formation between RAS and RAF and other protein kinases. Proc. Natl. Acad. Sci. USA 90:6213-6217.

12.Sittler, A., S. Walter, N. Wedemeyer, R. Hasenbank, E. Scherzinger, H. Eickhoff, G.P. Bates, H. Lehrach, and E.E. Wanker. 1998. SH3GL3 associates with the Huntingtin exon 1 protein and promotes the formation of polygln-containing protein aggregates. Mol. Cell 2:427-436.

13.Guldener, U., S. Heck, T. Fielder, J. Beinhauer, and J.H. Hegemann. 1996. A new efficient gene disruption cassette for repeated use in budding yeast. Nucleic Acids Res. 24:2519-2524.

14.Schiestl, R.H. and R.D. Gietz. 1989. High efficiency transformation of intact yeast cells using single stranded nucleic acids as a carrier. Curr. Genet. 16:339-346.

15.Goehler, H., M. Lalowski, U. Stelzl, S. Waelter, M. Stroedicke, U. Worm, A. Droege, K.S. Lindenberg, et al. 2004. A protein interaction network links GIT1, an enhancer of huntingtin aggregation, to Huntington's disease. Mol. Cell 15:853-865.

16.Huntington's Disease Collaborative Research Group. 1993. A novel gene containing a trinucleotide repeat that is expanded and unstable on Huntington's disease chromosomes. Cell 72:971-983.

17.Holbert, S., I. Denghien, T. Kiechle, A. Rosenblatt, C. Wellington, M.R. Hayden, R.L. Margolis, C.A. Ross, et al. 2001 The Gln-Ala repeat transcriptional activator CA150 interacts with huntingtin: neuropathologic and genetic evidence for a role in Huntington's disease pathogenesis. Proc. Natl. Acad. Sci. USA 98:1811-1816.

18.Miller, J.H. 1972. Assay of beta-galactosidase, p. 352-355. In Experiments in Molecular Biology. CSH Laboratory Press, Cold Spring Harbor, NY.

19.Kaiser, R. and G. Gottschalk. 1972 Elementary Tests for Data Analysis, p. 2-10. BI Hochschultaschenbücher, Mannheim.

Received 22 December 2004; accepted 11 May 2005.

Address correspondence to Sylvia Krobitsch, Max Planck Institute for Molecular Genetics, Ihnestrasse 73, 14195 Berlin, Germany.e-mail:krobitsc@molgen.mpg.de

To purchase reprints of this article, contact apfeffer@BioTechniques.com 